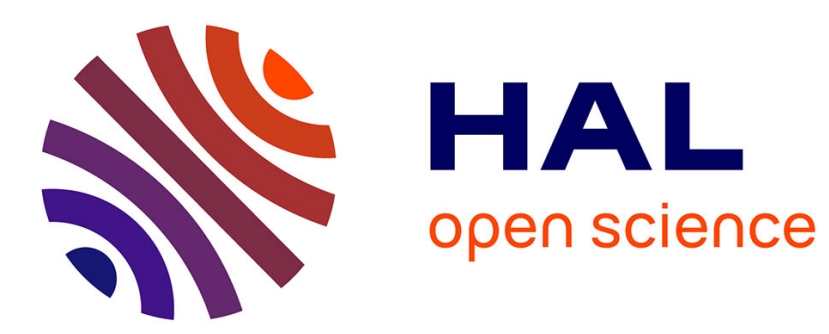

\title{
Computation of Hopf bifurcations coupling reduced order models and the asymptotic numerical method
}

\author{
J. Heyman, G. Girault, Y. Guevel, C. Allery, Aziz Hamdouni, Jean-Marc
}

Cadou

\section{- To cite this version:}

J. Heyman, G. Girault, Y. Guevel, C. Allery, Aziz Hamdouni, et al.. Computation of Hopf bifurcations coupling reduced order models and the asymptotic numerical method. Computers and Fluids, 2013, 76, pp.73-85. 10.1016/j.compfluid.2013.02.001 . hal-00985306

\section{HAL Id: hal-00985306 https://hal.science/hal-00985306}

Submitted on 23 Nov 2020

HAL is a multi-disciplinary open access archive for the deposit and dissemination of scientific research documents, whether they are published or not. The documents may come from teaching and research institutions in France or abroad, or from public or private research centers.
L'archive ouverte pluridisciplinaire HAL, est destinée au dépôt et à la diffusion de documents scientifiques de niveau recherche, publiés ou non, émanant des établissements d'enseignement et de recherche français ou étrangers, des laboratoires publics ou privés. 


\title{
Computation of Hopf bifurcations coupling reduced order models and the Asymptotic Numerical Method.
}

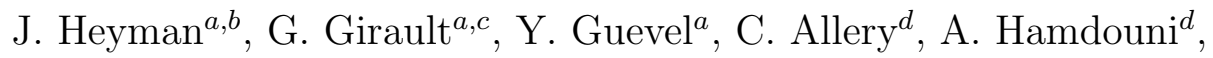 \\ J. M. Cadou ${ }^{a *}$ \\ ${ }^{a}$ Laboratoire d'Ingénierie des Matériaux de Bretagne, Université Européenne de \\ Bretagne, Université de Bretagne Sud, Rue de Saint Maudé, B.P. 92116, 56321 Lorient \\ Cedex - France \\ ${ }^{b}$ Current address: École Polytechnique Fédérale de Lausanne, Laboratoire dHydraulique \\ Environnementale, Station 18, 1015 Lausanne - Switzerland \\ ${ }^{c}$ Centre de recherche des Écoles de Saint-Cyr Coëtquidan, Écoles de Coëtquidan, 56381, \\ Guer cedex, France. \\ ${ }^{d}$ LEPTIAB, Université de la Rochelle, Pôle Science et Technologie, Avenue Michel \\ Crépeau, 17042 La Rochelle Cedex 1, France
}

\section{Abstract}

This work deals with the computation of Hopf bifurcation points in the framework of two-dimensional fluid flows. These bifurcation points are determined by using a Hybrid method[1] which associates an indicator curve and a Newton method. The indicator provides initial values for the Newton method. As the calculus of this indicator is time consuming, we suggest using an algorithm to save computational time. This algorithm alternates reduced order and full size step resolution which are all carried out by using a pertubation method. Hence, the computed vectors on the full size problem are used to define the reduced order model. As the low-dimensional model has a finite validity range, we propose a simple criterion which makes it possible to know

\footnotetext{
*J.M. Cadou, jean-marc.cadou@univ-ubs.fr
} 
when the basis has to be updated. The latter phase is carried out by going through a new full step which permits to build a new basis and, thus, compute a supplementary part of the indicator curve. Some numerical tests, such as the classical lid-driven cavity or the flow in a channel, permit to fix the optimum values of the parameters for the proposed method. The objective of this study is to save computational time without modifying the performance of the Hybrid method initially introduced in Ref.[1]. These numerical methods are applied to 2D fluid flows (flow in a channel and the 2D lid-driven cavity). Our conclusions, therefore, hold only for these kinds of problem.

Key words: Hopf bifurcation points, reduced order model, asymptotic numerical method, fluid mechanics,

\section{INTRODUCTION}

A Hopf's bifurcation is an instability of the fluid flow which is characterized by the transition from a stationary state towards an instationary one. From a mathematical point of view, a Hopf's bifurcation appears when a complex conjugate pair of eigenvalues of the linearized Jacobian matrix crosses the imaginary axis of the complex plane.

Usually, such instabilities are numerically computed by means of the socalled direct methods and the indirect ones with the monitoring of an indicator.

The principle of the direct method consists in iteratively computing the solution of a nonlinear algebraic system which corresponds to a Hopf bifur- 
cation point $[4,5]$. Unfortunately, the convergence of the algorithm depends on the choice of the initial value.

The indirect method is based on the track of an indicator which has the property of being null at a bifurcation point. The latter consists, for example, in computing the eigenvalues of the Jacobian matrix, and in determining when a pair of complex conjugated values crosses the imaginary axis, see references $\underline{[2,3]}$. In [7], Cadou et al. use an indirect method to determine the Hopf bifurcation for the 2D academic problems of the flow around a cylinder and the flow in a lid-driven cavity. In fact, they introduce a bifurcation indicator which has the property of being null at the singular points. To avoid large computing times, the computation of this bifurcation indicator is done with a perturbation method. Whereas it gives accurate values of bifurcation points, this method requires a lot of calculi, and is not automatic.

To circumvent this drawback, Brezillon et al.[1] propose to couple two methods (direct and indirect ones) resulting in a hybrid algorithm. The idea of this hybrid method is that the initial guesses of the Newton algorithm are determined by an indicator calculation. The numerical results show that the indicator calculation provides several initial values but some candidates do not lead to the convergence of the Newton's algorithm, resulting in a large amount of CPU time without ensuring the convergence of the hybrid method.

Recently, Girault et al.[8] propose to improve the robustness of the hybrid method by automatically determining the minima of the indicator curve, and using them as initial values for the Newton algorithm. In the case 
of the 2D lid driven cavity, the results show that the method is efficient. Nevertheless, this algorithm still requires long CPU times mainly dedicated to the calculation of the indicator because of the size of the manipulated Jacobian matrix. Previous studies, $[1,8]$, have shown that the calculation of the indicator is the step that consumes the most CPU time in the hybrid method. The idea of the present sudy is to propose an efficient numerical method allowing for a faster calculation of the indicator.

A means of decreasing these CPU times is either the use of a specific linear solver $[9,10]$ or that of reduced order models. The main objective of such methods is to replace a large fully dicretized model with a reduced model describing correctly the dynamic behavior and preserving fundamental properties of the full model. Hence, the choice of the reduction technique is important. Today, the POD is one of the most widely used techniques of model order reduction. Introduced for the first time by Lumley[11] for the study of turbulent flows, the POD consists in a linear decomposition yielding a physical and orthogonal spatial basis in which dimensions are lower than the initial model. The reduced order models are then obtained by projecting the full model onto this POD basis. The main drawback of the POD is that it requires computations of the unkown fields, in the full size problem, to build the snapshots needed for determination of the reduced basis. This approach was used by Cazemier et al. [12] to compute Hopf bifurcations in 2D lid-driven cavities. They carry out a first time-dependent simulation of the Navier-Stokes equations for a large Reynolds number. Next, they 
use these results to build, with the POD, a reduced eigenvalues problem in order to determine precise Hopf bifurcation points. In the case of the hybrid method, this reduction technique is not the best way to proceed. Indeed, as shown in Ref. [8], a single computation of the indicator can provide the Newton method with a lot of initial guesses, which can lead sometimes to 4 or 5 Hopf bifurcation points. A POD analysis can then be performed using this first computation although results will probably be the same as the ones obtained with the initial calculi. So, the benefit of using a reduced model is nullified by the fact that it requires a first computation which can be time consuming and not useful for the determination of bifurcation points.

A reduced technique has been recently proposed in [13] and applied to define a linear solver[10] or to study nonlinear vibrations of plates[14]. In this technique, the vectors computed in the first steps of the perturbation method are used to build a basis which permits to determine the other part of the nonlinear solution. In the case of the linear solver, a preconditioning technique is added to the reduction method with a view to avoiding a lot of basis modifications. It means that the basis is the same for almost all the computations. In the framework of nonlinear vibrations of plates[14], as the nonlinear curves do not evolve a lot all through the computations, a single basis computation is necessary to compute almost all the nonlinear solution curves. For the hybrid method, as shown in references $\underline{[1,} \underline{8]}$, the indicator curves which depend on the angular frequency are very nonlinear. A single basis evaluation is therefore not sufficient to determine the entire response 
indicator curve. So the basis has to be upgraded all along the indicator computation. We propose to alternate between full size problem resolution and reduced order models steps. A full size computation is performed when the reduced order solutions do not verify a simple residual criterion. With this full size computation, a new basis is defined and permits then to carry out additional calculi of the indicator on the reduced order problem. As the computation on the full size model is very time consuming, mainly due to the fine spatial discretization, the point is to limit these full size computational steps.

The paper is organized as follows. Section 2 is devoted to theoretical aspects recalling the governing equations for an incompressible viscous flow, and the stability analysis. Section 3 presents the model reduction technique. Some numerical results related to the academic problems of the lid-driven cavity and the flow in a channel are given in section 4, and show the relevancy of the proposed method.

\section{Elements of theory}

\subsection{Governing equations}

In this study, we consider the movement of a viscous incompressible flow described by the following Navier Stokes equations:

$$
\begin{gathered}
\frac{\partial \mathbf{u}}{\partial \mathrm{t}}-\nu \nabla^{2} \mathbf{u}+\mathbf{u} \cdot \nabla \mathbf{u}+\frac{\nabla \cdot p}{\rho}=0 \quad \text { in } \\
\nabla \cdot \mathbf{u}=0 \quad \text { in } \quad(\Omega)
\end{gathered}
$$




$$
\mathbf{u}=\lambda \mathbf{u}_{d} \quad \text { on } \quad\left(\partial_{\mathbf{u}} \Omega\right)
$$

In these equations, $\Omega$ and $\partial_{\mathbf{u}} \Omega$ are the fluid domain and the boundary surface where velocity is imposed. The symbols $\mathbf{u}, p, \rho, \nu$ stand, respectively, for the velocity field, the pressure, the density and the kinematic viscosity of the fluid. The boundary condition imposes a velocity field of intensity $\lambda$ which is linked to the Reynolds Number defined by $R e=\lambda\left|\mathbf{u}_{d}\right| L / \nu$ with $L$ being a geometrical reference length. For each numerical example, this Reynolds number will be precisely defined and is the bifurcation parameter used in this study.

The weak formulation associated to equations [17] is written:

$$
M(\dot{\mathbf{U}})+L(\mathbf{U})+Q(\mathbf{U}, \mathbf{U})-\lambda F=0
$$

where $M$ is the mass matrix, $L$ and $Q$ are linear and quadratic operators: $L$ contains the pressure and the diffusion terms while the convective terms are contained in $Q$. The vector $\mathbf{U}$ is a concatenated vector composed of the velocity $\mathbf{u}$ and the pressure $p$. The term $\lambda F$ can be considered as an external load vector created by the boundary condition on $\partial_{\mathbf{u}} \Omega$. One can refer to $[17]$ for a complete presentation of all these operators.

\subsection{Stability analysis}

The stability of the flow is studied by introducing a perturbation $\Delta U(\mathbf{x}, t)$ of the stationary term $U^{S}$. This perturbation can be considered as a product of the spatial term $V(\mathbf{x})$ by the temporal term $e^{i \omega t}$ : 


$$
\Delta U(\mathbf{x}, t)=V(\mathbf{x}) e^{i \omega t}
$$

In expression (5), $\omega$ designates the pulsation of the periodic flow, and $V(\mathbf{x})$ stands for the complex mode of perturbation.

Introducing expression (5) into equations (4), and neglecting quadratic and higher order terms in $V$, it becomes the following linear system:

$$
\left\{\begin{array}{l}
L\left(U^{S}\right)+Q\left(U^{S}, U^{S}\right)-\lambda F^{S}=0 \text { in } \Omega \\
L(V)+Q\left(V, U^{S}\right)+Q\left(U^{S}, V\right)+i \omega M(V)=0 \text { in } \Omega \\
V_{\mathbf{u}}=0 \text { on } \partial_{\mathbf{u}} \Omega
\end{array}\right.
$$

A Hopf's bifurcation corresponds to a vector $\Lambda=\left\{U^{S}, V, \lambda, \omega\right\}$ which is the solution to equations (6). In the latter equations, $V_{\mathbf{u}}$ stands for the velocity part of the concatenated vector $V$ on the boundary $\partial_{\mathbf{u}} \Omega$ where a velocity $\mathbf{u}_{d}$ is imposed. Finally, the previous system is written under the following form:

$$
R(\Lambda)=0 \text { in } \Omega
$$

\subsection{Bifurcation indicator}

This section is devoted to the presentation of the bifurcation indicator and of the asymptotic numerical method applied to the search of Hopf's 
bifurcation points. All the elements have already been presented in $[\underline{7,} \underline{8]}$ and the main ideas are outlined in this paper.

Firstly, the stationary solution $U^{S}$ is determined by using an asymptotic numerical method according to the algorithm introduced by $\underline{[18]}$ and applied in a framework of fluid mechanics[17]. This method, denoted by ANM, consists in the association of a perturbation method and a discretization technique, the finite element method in our case. Let us note that when a singular point occurs in the nonlinear solution branch, for example a steady bifurcation point, then a specific ANM has to be used (see Ref. [21]). Nevertheless, for the most part of the computations, a standard ANM is used, such as the one presented in Ref. [18]. ANM can be coupled with correction steps (see for example [19]) when the obtained accuracy is not satisfactory. Secondly, equations (6) are rewritten assuming that a perturbation is created by a random force vector $f$ of intensity $\phi$, and introducing the tangent operator $L_{t}=L(\bullet)+Q\left(\bullet, U^{S}\right)+Q\left(U^{S}, \bullet\right)$ :

$$
\begin{gathered}
L_{t}(V)+i \omega M(V)=\phi f \\
V_{\mathbf{u}}=0 \quad \text { on } \quad \partial_{\mathbf{u}} \Omega
\end{gathered}
$$

In the previous equations, the scalar $\phi$ is our bifurcation indicator. A Hopf bifurcation corresponds to a null value of $\phi$. The same kind of indicator is introduced to compute stationary bifurcation $[7,21]$. The bifurcation indicator, $\phi$, is computed for a fixed and known stationary solution $\left(U^{S}, \lambda\right)$ (the 
tangent operator explicitly depending on $\left.U^{S}\right)$.

Then, $V^{R}$ and $V^{I}$ are introduced as the real and imaginary parts of the complex vector $V$. The equations are discretized according to the classical finite element method, using the velocity formulation and a penalty method on the discrete continuity equation (see Ref. [17]). Thus, the operator $K_{t}$ is the discrete form of $L_{t}$, and the following discrete algebraic equations have to be solved:

$$
\left[\begin{array}{cc}
K_{t} & -\omega M \\
\omega M & K_{t}
\end{array}\right]\left\{\begin{array}{c}
V^{R} \\
V^{I}
\end{array}\right\}=\left\{\begin{array}{c}
\phi f \\
0
\end{array}\right\} \quad \text { in } \Omega
$$

With the boundary condition:

$$
V_{\mathbf{u}}=0 \quad \text { on } \quad \partial_{\mathbf{u}} \Omega
$$

In order to have a well-posed problem and avoid numerical instabilities [7], the following quadratic condition is considered:

$$
\|V\|^{2}=\left\|W_{0}\right\|^{2}
$$

where $\|\bullet\|$ is the Euclidian norm of the vector $\bullet$. The vector $W_{0}$ stands for the solution of equation (10) with $\phi=1$ and $\omega=0$.

Equation (10) is solved using the asymptotic numerical method. From a known and singular solution $\left\{V_{0}, \omega_{0}, \phi_{0}\right\}$, the pulsation $\omega$ is defined as the sum of an initial value $\omega_{0}$ and a path parameter $\tilde{\omega}$. The unknowns $\phi, V^{R}$ 
and $V^{I}$ are then sought as polynomial expansions of the path parameter:

$$
\left\{\begin{array}{c}
\phi \\
V^{R} \\
V^{I}
\end{array}\right\}=\left\{\begin{array}{c}
\phi_{0} \\
V_{0}^{R} \\
V_{0}^{I}
\end{array}\right\}+\tilde{\omega}\left\{\begin{array}{c}
\phi_{1} \\
V_{1}^{R} \\
V_{1}^{I}
\end{array}\right\}+\ldots+\tilde{\omega}^{p}\left\{\begin{array}{c}
\phi_{p} \\
V_{p}^{R} \\
V_{p}^{I}
\end{array}\right\}
$$

The previous expressions (13) are inserted into equations (10), (11) and(12).

Equating like powers of $\tilde{\omega}$, the following set of linear equations is obtained:

Order 0 in $\tilde{\omega}$ :

$$
\left\{\begin{array}{l}
{\left[\begin{array}{cc}
K_{t} & -\omega_{0} M \\
\omega_{0} M & K_{t}
\end{array}\right]\left\{\begin{array}{c}
V_{0}^{R} \\
V_{0}^{I}
\end{array}\right\}=\left\{\begin{array}{c}
\phi_{0} f \\
0
\end{array}\right\} \text { in } \Omega} \\
V_{0}^{R}=V_{0}^{I}=0 \text { on } \partial \mathbf{u} \Omega \\
\left\|V_{0}\right\|^{2}=\left\|W_{0}\right\|^{2} \text { in } \Omega
\end{array}\right.
$$


Order $p$ in $\tilde{\omega}$ :

$$
\left\{\begin{array}{l}
{\left[\begin{array}{cc}
K_{t} & -\omega_{0} M \\
\omega_{0} M & K_{t}
\end{array}\right]\left\{\begin{array}{c}
V_{p}^{R} \\
V_{p}^{I}
\end{array}\right\}=\left\{\begin{array}{c}
\phi_{p} f+M\left(V_{p-1}^{I}\right) \\
-M\left(V_{p-1}^{R}\right)
\end{array}\right\} \text { in } \Omega} \\
V_{p}^{R}=V_{p}^{I}=0 \text { on } \partial \mathbf{u} \Omega \\
2\left\langle V_{p}, V_{0}\right\rangle+\sum_{r=1}^{p-1}\left\langle V_{p-r}, V_{r}\right\rangle=0 \text { in } \Omega
\end{array}\right.
$$

where the symbol $\langle\bullet, \bullet\rangle$ stands for the inner product.

Finally, the initial nonlinear problem defined by expressions (10), (11) and(12) is transformed into a sequence of linear systems (14) and (15) having all the same discrete tangent operator with different right hand sides. For all the previous linear problems, a single matrix triangulation and $\mathrm{p}$ backward/forward substitutions allow for the computation of the unknown $\left\{V_{p}^{R}, V_{p}^{I}, \phi_{p}\right\}$. Due to a lower validity range, the polynomial expansions are replaced with rational ones, using Padé Approximants[22]. These Padé Approximants are defined by:

$$
\left\{\begin{array}{c}
\phi \\
V^{R} \\
V^{I}
\end{array}\right\}=\left\{\begin{array}{c}
\phi_{0} \\
V_{0}^{R} \\
V_{0}^{I}
\end{array}\right\}+\sum_{i=1}^{p-1} f_{i}(\tilde{\omega}) \tilde{\omega}^{i}\left\{\begin{array}{c}
\phi_{i} \\
V_{i}^{R} \\
V_{i}^{I}
\end{array}\right\}
$$


where $f_{i}(\tilde{\omega})$ are rational functions of the path parameter with the same denominator. The validity range of the approximants is evaluated using the following expression according to a small user parameter $\delta$ :

$$
\delta=\frac{\left\|V_{p}\left(\tilde{\omega}_{\text {MaxPadé }}\right)-V_{p-1}\left(\tilde{\omega}_{\text {MaxPadé }}\right)\right\|}{\left\|V_{p}\left(\tilde{\omega}_{\text {MaxPadé }}\right)-V_{0}\right\|}
$$

Once the maximum value $\tilde{\omega}$ of the path parameter is computed, it is introduced in the rational expressions (16) thereby defining a new starting point $\left(\omega_{0}, V_{0}^{R}, V_{0}^{I}, \phi_{0}\right)$. This defines the so-called continuation method $\underline{[23]}$ and permits the computation, in a step-by-step manner, of the indicator for a chosen range of the path parameter $\omega$.

Finally, for each chosen stationary solution, $U^{S}$, the indicator curve $\phi(\omega)$ is explicitely known by using the previous equations. Finding a Hopf bifurcation then consists in determining the solution $U^{S}$ where the indicator is null. This implies a lot of indicator calculi, and is not very efficient compared to other classical methods of determining Hopf bifurcation. Nevertheless, as these indicator curves are computed by using a perturbation method (ANM), this leads to a total computational time which is lower than the one obtained by using, for example, a classical incremental-iterative method. As already mentioned, Brezillon et al. $[1]$ and Girault et al. $\underline{[8]}$, in order to propose an efficient algorithm to detect Hopf bifurcation, have introduced a Hybrid method which consists in the association of the previous indicator with a Newton iterative scheme. 


\subsection{Hybrid method}

The hybrid method, first introduced in [1], allows the computation of multiple Hopf bifurcations $[8]$ by coupling the Newton method for the iterative resolution of the nonlinear problem with a calculation of the indicator $\phi(\tilde{\omega})$.

The robustness of the hybrid algorithm is based on the choice of the initial guesses for the Newton algorithm. Thus, numerical results show that choosing the vector $\left\{\omega, V^{R}, V^{I}\right\}$ evaluated for all the minima of the indicator gives multiple initial vectors for which convergence of the Newton algorithm is ensured even for Reynolds Number and Strouhal numbers far from their critical values.

The minimina of the indicator are determined classically by computing the roots of the first derivative functions $d \phi / d \tilde{\omega}$, and by checking the sign of the second derivative $d^{2} \phi / d \tilde{\omega}^{2}$ with respect to the path parameter $\tilde{\omega}$. These roots are then introduced as initial guesses of the Newton iterative scheme. The hybrid method can thus be summarized by the followings two steps.

Step 1: Indicator computations for a fixed Reynolds number $\left(U_{0}^{S}, \lambda_{0}\right.$ fixed).

- For $\omega \in\left[0, \omega_{\max }\right]$ with $\omega_{\max }$ chosen by the user

$-\omega=\omega_{0}+\tilde{\omega}$

- Indicator calculus, $\phi$, with expressions (14), (15) and (16)

- Determination of the minimum of the function $\phi(\tilde{\omega})$ for $\tilde{\omega}=\tilde{\omega}_{m i n i}^{m}$

- Compute $\phi_{0}^{m}, V_{0}^{R, m}$ and $V_{0}^{I, m}$ by replacing $\tilde{\omega}=\tilde{\omega}_{m i n i}^{m}$ in Eq. (16) 
- Save $\Lambda_{0}^{m}=\left\{U_{0}^{S}, V_{0}^{m}, \lambda_{0}, \omega_{m i n i}^{m}=\omega_{0}+\tilde{\omega}_{m i n i}^{m}\right\}$

- Continuation method up to $\omega_{0} \geq \omega_{\max }$ with $\omega_{0}=\omega_{0}+\tilde{\omega}_{\text {MaxPadé }}$ (Eq. 17).

Step 2: Newton method

- For $m=1, M d o$

- Initial value $\Lambda_{0}^{m}=\left\{U_{0}^{S}, V_{0}^{m}, \lambda_{0}, \omega_{\text {mini }}^{m}\right\}$

- Newton iterations

$$
\Lambda_{i}^{m}=\Lambda_{i-1}^{m}+\Delta \Lambda^{m}
$$

- With the increment $\Delta \Lambda^{m}$ solution

$\left.\frac{\partial R}{\partial \Lambda}\right|_{\Lambda_{i-1}^{m}} \Delta \Lambda^{m}=-R^{m}\left(\Lambda_{i}\right)$

With $\left.\frac{\partial R}{\partial \Lambda}\right|_{\Lambda_{i-1}}$ is the Jacobian matrix

Convergence if $\left\|R\left(\Lambda_{i}^{m}\right)\right\| \leq \eta$ ( $\eta$ being an user tolerance)

Finally, the two previous steps define the hybrid method, and a Hopf bifurcation point is found when $\left\|R\left(\Lambda_{i}^{m}\right)\right\| \leq \eta$. According to previous studies [8], it is the first step which takes the most time. It is this step for which we propose to use a reduction technique

\section{Model Reduction Technique}

This section is devoted to the presentation of the model reduction techniques used in this study. It is noticed that such technique allows to reduce 
the size of the problem by projecting the equations on a reduced basis. Moreover, the reduction must preserve the properties of the initial model. In this study, the reduction is applied after the linearization by the ANM and the FEM discretization especially on the linear systems (14) and (15). The proposed reduction technique is based on the ANM for the generation of the basis vectors. As mentioned above, the proposed reduction technique has already been used in nonlinear thin shell analysis (Ref. [13]) or nonlinear vibrations framework (Ref. [14]), or to define linear solvers (Ref. [10]). As this reduction order gave very interesting results in previous works, it has been adapted and applied to the computation of the bifurcation indicator.

\subsection{General overview of the projection technique}

The principle of the model reduction is based on the projection of the unknowns onto a vector space of reduced dimensions as follows:

$$
\mathbf{V}=\Psi \cdot \mathbf{v}
$$

where $\mathbf{v}$ and $\mathbf{V}$ stand, respectively, for the reduced and the complete unkonwn vector of size nred and $N T$, with nred $\ll N T$. In this numerical study, NT is the number of d.o.f. of considered meshes. The operator $\Psi$ stands for the projection matrix of dimension $N T \times$ nred .

Whatever the method considered, one has to solve an algebraic system of great size which can be formulated as follow: 


$$
\mathbf{K} \cdot \mathbf{V}=\mathbf{F}
$$

In this study, the matrix $\mathbf{K}$, the right-hand side $\mathbf{F}$ and the unknown vector $\mathbf{V}$ are the ones defined at each order $p$ of the indicator computation (eq. (15)). Using definition (18) in equation (19), it becomes:

$$
\mathbf{K} \cdot \Psi \cdot \mathbf{v}=\mathbf{F}
$$

Multiplying both sides of equation (20) by the transpose of $\Psi$, noted ${ }^{t} \Psi$, it results in:

$$
{ }^{t} \Psi \cdot \mathbf{K} \cdot \Psi \cdot \mathbf{v}={ }^{t} \Psi \cdot \mathbf{F}
$$

Then, one has to solve the following problem of reduced dimensions nred $\times$ nred:

$$
\mathcal{K} \cdot \mathbf{v}=\mathcal{F}
$$

where $\mathcal{K}$ and $\mathcal{F}$ stand for the reduced forms of $\mathbf{K}$ and $\mathbf{F}$.

\subsection{Application to the indicator computation}

The key point of the reduced order methods is the choice of the projection operator $\Psi$. In this work, this operator is build by the vectors computed on the full size problem at the ANM step $j$. These vectors are solutions to problem (15) at step $j$ of the computation of the indicator. 
The method that is proposed in order to define the projection operator consists in not differentiating between the real and the imaginary part of the reduced unknown vector $\mathbf{v}$. Hence, the operator $\Psi$ is defined as follows:

$$
\Psi=\left\{\begin{array}{ccc}
V_{1}^{R, \perp}, & \ldots, & V_{\text {nred }}^{R, \perp} \\
V_{1}^{I, \perp}, & \ldots, & V_{\text {nred }}^{I, \perp}
\end{array}\right\}
$$

where nred stands for the number of vectors of the reduced basis and $\perp$ for the vectors which have been orthonormalized. The latter step is a necessary towards building the Padé approximants [24] used in the indicator computation. Applying this methodology to the equations issued from ANM (eq. (15)), one has to solve $p+1$ linear problems of reduced dimensions, defined by, for example at order $p$ :

$$
\left\{\begin{array}{c}
\left\{\mathcal{K}_{t}\right\}\left\{\mathbf{v}_{p}\right\}={ }^{t} \Psi\left\{\begin{array}{c}
\phi_{p} F+M\left(\mathbf{V}_{p-1}^{I}\right) \\
-M\left(\mathbf{V}_{p-1}^{R}\right)
\end{array}\right\} \\
2\left\langle\mathbf{v}_{p}, \mathbf{v}_{0}\right\rangle+\sum_{r=1}^{p-1}\left\langle\mathbf{v}_{p-r}, \mathbf{v}_{r}\right\rangle=0
\end{array}\right.
$$

where the reduced matrix $\mathcal{K}_{t}$ is defined by the following expression:

$$
\left\{\mathcal{K}_{t}\right\}={ }^{t} \Psi\left[\begin{array}{cc}
K_{t} & -\omega_{0} M \\
\omega_{0} & K_{t}
\end{array}\right] \Psi
$$


Finally, with this reduction technique, only one reduced matrix has to be built and has the size nred $\times$ nred.

During the computations, one has to be sure that the reduced solutions provide the same minima for the indicator as the ones obtained in the case of the full size resolution.Indeed, the computations carried out with the reduced order model can yield solutions which are not satisfactory. To make sure that the solutions given by the reduced and the full size models are close, we propose an accuracy criterion which is based on the following full discrete residual vector:

$$
\left\{\begin{array}{c}
R^{R} \\
R^{I}
\end{array}\right\}=\left\{\begin{array}{c}
K_{t} \cdot V^{R}-\omega M \cdot V^{I}-\phi f \\
\omega M \cdot V^{R}+K_{t} \cdot V^{I}
\end{array}\right\}
$$

The indicator computations are therefore performed using the reduced model as long as the following criterion is satisfied:

$$
\frac{\left\|R^{R}\right\|}{\left\|\phi_{f}\right\|}<\epsilon \text { and } \frac{\left\|R^{I}\right\|}{\left\|\phi_{f}\right\|}<\epsilon
$$

where $\|\bullet\|$ represents the Euclidian norm and $\epsilon$ is a chosen tolerance paramater.

Once the criterion (27) is reached, the following step is taken by solving the full size problem (Eq. 14 and 15). In this way, the basis (relation (23)) is then automatically upgraded by the new vectors computed on the full size problem. The following part of the indicator curve is then computed 
by the reduced technique until the criterion (Eq. 27) is reached one more time.Ultimately, the proposed algorithm consists in alternating between full size steps and reduced steps, with a view to minimizing the number of full steps.

\subsection{Algorithm}

The proposed numerical method for the computation of the indicator bifurcation can be summarized by the following algorithm:

1. Computation of the steady solution $U^{S}$ (Re fixed)

2. Bifurcation indicator, $\omega_{0}=0$, Ordre P fixed, $\omega_{\max }$ chosen.

2.1 Full size problem

2.2 Solve Eq. (14) and (15)

2.3 Validity range of the Padé approximants, $\tilde{\omega}_{\text {MaxPadé }}$ (Eq. 17)

$2.4 \omega_{0}=\omega_{0}+\tilde{\omega}_{M a x P a d e ́}$

2.5 If $\left(\omega_{0}>\omega_{\max }\right)$ go to 3

2.5 Save the vectors $V_{p}^{R, \perp}$ and $V_{p}^{I, \perp}$

2.6 Reduction phase

2.7 Solve Eq. (24) from $p=1$ to $P$

2.8 Validity range of the Padé approximants, $\tilde{\omega}_{\text {MaxPadé }}(E q .17)$

$2.9 \omega_{0}=\omega_{0}+\tilde{\omega}_{\text {MaxPadé }}$

2.10 If $\left(\omega_{0}>\omega_{\max }\right)$ go to 3 
2.11 If criterion (27) is satisfied goto 2.6 else goto 2.1

3. Hybrid method (see section 2.4).

This algorithm is numerically evaluated in the following section.

\section{Numerical results}

\subsection{Numerical tests}

Two numerical tests are considered in this section. Spatial discretization for both examples are performed by using the classical finite element method. The chosen finite element is a quadrilateral element, with 9 nodes for the velocity (bi-quadratic interpolation) and 3 for the pressure (linear interpolation) [20]. The continuity equation is solved by using a penalty method $[20]$. For both examples, the stationary solutions are computed using the Asymptotic Numerical Method defined for the Navier-Stokes equation in Ref. [17].

The first example is the classical lid-driven cavity with a length and a width equal to 1.Parameter $\mathrm{A}$, which defines the aspect ratio, is then equal to 1 (see Fig. 1). The number of nodes of the mesh is equal to 6561 (i.e. 13.122 unknowns) and is the same as the ones used in Ref. [1]. A lot of critical Reynolds numbers are available in the literature for this example. Table 1 presents some of those critical Reynolds numbers and Strouhal numbers computed by several authors. For this example the Strouhal number is 
determined by the following expression:

$$
S t=\frac{D \cdot \omega}{2 \pi u}
$$

where $\mathrm{D}$ is the width of the cavity (equal to 1.0) and $u$ designates the imposed velocity in the upper side of the cavity. For this example the first Hopf bifurcation appears for a Reynolds number close to 8000 and a Strouhal number near 0.45 . This first example allows us to assess the proposed reduction technique and to give a validity range of the two algorithm parameters which are the number of reduced vectors, nred, and the value of the accuracy parameter, $\epsilon$, of the criterion in Eq. (27).

The second example deals with the flow in a channel with a suddenly expanded part (see Fig. 2), initially studied by Mizushima et al. in Ref. [25] and is quite interesting for stability studies. In fact this example exhibits two stationary bifurcations before a third instability which corresponds to a Hopf bifurcation. These two stationary bifurcation points are shown in Fig. 3 where the velocity at point P1 (defined in Fig. 2) is plotted versus the Reynolds number of the flow between 0 and 70. This Reynolds number is defined by:

$$
R e=\frac{u_{\max } h}{2 \nu}
$$

where $h$ (here $\mathrm{h}=2$ ) is the height of the entrance channel and $u_{\max }$ is the maximum velocity of the parabolic profile imposed on the entrance. The 
first stationary bifurcation point (where the flow becomes asymmetric) is equal to 48.74. The second bifurcation point (where the flow becomes symmetric again) is 65.1. These critical points are also obtained by using a bifurcation indicator which has been first introduced in Ref. [7], and used to study the Coanda effect[34]. This stationary indicator has been improved in Ref. $[21]$ where a numerical method is introduced to follow the stationary bifurcating solutions emanating from the singular points. The method has been used in this study to compute all the stationary solutions plotted in Fig. 3, and notably the symmetric solution after the second bifurcation point where the Hopf bifurcation point is searched. For a comparison of the computational times, 4 meshes are used in this second example, from 32.322 d.o.f. to nearly 350.000 d.o.f..The results for these meshes are summarized in Table 2. The same table also gives the basic computational times for the numerical methods evaluated in this study. For example, the CPU times needed for a single eigenvalue computation (for a fixed Reynolds) are given for each studied mesh. The critical numbers for the Hopf bifurcation points are given in Table 3. The critical numbers obtained with ARPACK (Ref. [33]) and those given in Ref. [25] are also indicated in this table.

The Strouhal number for this example is determined by using the following expression:

$$
S t=\frac{h \cdot \omega}{4 \pi u_{\max }}
$$

The critical numbers found in this study are close to 1000 and 0.075 respec- 
tively for the Reynolds and for the Strouhal numbers (for the first bifurcation). Concerning the Strouhal number, which is close to 0.074 (see Table 3), our results agree well with the values given in Ref. [25]. The critical Reynolds number is greater than the one given in Ref. [25] $\left(\operatorname{Re}_{c} \approx 843\right)$. Nevertheless, our critical values are confirmed by several eigenvalue computations. These eigenvalues are plotted on the complex plane in Figures (4) for several values of the Reynolds number. These figures show that two conjugated complex eigenvalues cross the imaginary axis for a Reynolds value between 965 and 1023, and two cross the imaginary axis for a Reynolds value included in the range $[1559,1602]$. The angular frequency is approximately 47 for the first bifurcation point (see Figures 5-a and 5-b) and 100 for the second one (see Figures 5 -c and 5-d). To explain the difference between the results that are presented and those given in Ref. [25], the evolution of the first critical Reynolds number versus the number of unknowns has been plotted in Fig. (5). This curve shows that, for approximately the same number of unknowns (close to 10.000), the Hybrid method gives nearly the same critical Reynolds number as the one found by Mizushima et al. in Ref. [25]. We have plotted in Figures (6) the streamlines of the solutions (steady solution, real and imaginary parts of the eigenmode) at the first bifurcation point $\left(\operatorname{Re}_{c 1}=856\right.$ and $\left.\omega_{c 1}=40.29\right)$ carried out with a number of unknowns equal to 13000 . These figures agree well with the results presented in Ref. [25]. Moreover, as shown in Fig. (5), the number of unknowns required to obtain the convergence to an acceptable critical Reynolds number must be greater than 50000. This 
means that the mesh used in Ref. [25] is too coarse to obtain an accurate value of the critical Reynolds number.

\subsection{Results with the Hybrid method}

For this paper to be self-contained, it could be useful to recall the results obtained with the Hybrid method put forward in Ref. [1] and [8]. To this end, we propose to study the first example: the lid-driven cavity. As the first critical Reynolds number is close to 8000, the indicator $\phi$ is then plotted against the angular frequency for a Reynolds number equal to 8089 (see Fig. 7).Let us note that, in Ref. [8], the first bifurcation point is found with an indicator curve computed for a Reynolds number close to 2000. Nevertheless, from the curve plotted in Fig. 7, one can determine several initial values for the Newton method detailed in section (2.4).

These initial values are given in Table 4 . From the indicator curve, we have determined 9 initial values that are introduced in the Newton iterative scheme. From these 9 initial guesses, 6 values converge to a Hopf bifurcation point. All these singular points are different. Some of them are far from the Reynolds number for which the indicator curve is computed. The single indicator curve of Fig. 7 then permits to automatically compute 6 critical Reynolds and Strouhal numbers. From the results presented in Ref. [8], this curve is obtained with 66 steps of the continuation method defined in section (2.3) with an order of truncature of the asymptotic expansions equal to 30, and a parameter $\delta=10^{-6}$ (Eq. 17). The indicator curve is computed for an 
angular frequency in the range $[0,4100]$. Let us recall that each step in this continuation technique requires a matrix triangulation, and 'p' backward and forward substitutions. According to the analysis proposed in Ref. [8], the more consuming times, for the hybrid method, concern this matrix triangulation. The aim of the proposed numerical method is then to decrease these computational times by using reduced order models without modifying the results of the Hybrid method. For example, the number of initial values to be introduced in the Newton scheme must be the same with or without the reduction technique.

\subsection{Results with the proposed reduction technique}

For the first results concerning the reduction technique, we study the liddriven cavity. The indicator curve is computed for the same initial Reynolds number as previously $(\mathrm{Re}=8089)$. The proposed reduced model contains two parameters: the number of vectors, nred, to define the projection operator $\Psi$, and the accuracy $\epsilon$ which permits to check the validity of the reduced solutions. Table 5 shows the results obtained with the reduced technique. In this table, the number of vectors of the reduced technique vary between 10 and 60 , and the accuracy parameter is included in $\left[10^{-2}, 10^{2}\right]$. The results of the full model when the order of truncature varies between 20 and 30 are also given. So is the number of steps needed for each numerical method to compute the indicator curve up to a maximum value of the angular frequency equal to 4100. This number is denoted by N1 in Table 5. For all the 
computations, the parameter $\delta$, which governs the validity range of the Padé approximants (Eq. 17), is fixed and is equal to $10^{-6}$.

The first interesting result from this table is that the number of steps in the reduced technique is almost greater than the one needed in the full model. The table also shows that approximately the same number of Hopf bifurcation points (indicated by the symbol 'NH' in Table 5) can be obtained with the reduced technique as with the full model. The last column in Table 5 displays the number of singular values (see Table 4) determined with the full or the reduced technique. In Table 5, the symbol 'All' means that the six singular values given in Table 4 have been determined. Hence, out of the 29 indicator calculi presented in Table 5 carried out with RANM, 16 lead to the same results as those obtained with the full model. 6 calculi give all the 6 singular values but some of them are computed several times. 3 calculi are needed to determine the first 5 Hopf bifurcation points. Only 4 indicator computations give either no bifurcation points or few singular values (less than 3). These 4 indicator calculi require the smallest number of steps. Therefore, the results summarized in Table 5 show that the two parameters of the reduced technique have to be carefully chosen. For example, a great value of $\epsilon\left(\epsilon=10^{0}\right.$ or $\left.\epsilon=10^{1}\right)$ must be associated with a number of vectors, nred, greater than 30. Contrariwise, a small value of nred, for example nred $=10$, requires a small value of the accuracy parameter $\epsilon\left(\epsilon \leq 10^{-1}\right)$.

For a more precise view of the influence of these parameters on the computation of the indicator curve $\phi$,the curve obtained with the full model 
resolution (denoted by 'Reference' on these figures) as well as the ones obtained with the reduced order model (denoted by 'RANM') have been plotted in Fig. 7 and 8

In Fig. 7, the RANM parameters are nred $=60$ and $\epsilon=10^{\circ}$. For these values, according to the results shown in Table 5, the RANM and the full model lead to the same number of Hopf bifurcation points $(\mathrm{NH}=6$ in Table 5) and the same number of Newton iterations. This is confirmed by the results plotted in Fig. 7, where the curve obtained with RANM exactly follows the curve obtained with the full model. For Fig. 8, where the RANM parameters $\epsilon$ and nred are respectively equal to $10^{2}$ and 60 , some parts of the curve obtained with RANM are totally different from the reference curve. Moreover, for some values of the angular frequency, the solution obtained with RANM jumps to another part of the curve without passing through the minimum of the curve (for an angular pulsation close to 3500 for example).

The last interesting feature of the results presented in Table 5 deals with the number of full steps during the resolution with the RANM. This number is given in Table 5, and is denoted by 'NT'. If we only analyze the reduced calculi which give the same bifurcation points as the full model ('All' in the last column), the number of full steps is between 13 and 24. Finally compared to the full model (66 steps), the number of full steps gained with the reduction technique is approximately between 60 to $80 \%$.

This minimum value of full steps (i.e. the number of times where the reduced basis is upgraded) is relatively great compared to the results obtained 
in Ref. [14] where the same technique is used in the framework of nonlinear vibration. Indeed, in this latter study, a single full step is necessary to determine the entire nonlinear solution. Nevertheless, the solution in the vibration problem does not evolve as much as it does in the present study. In fact the indicator curves (see Fig. 7) are strongly nonlinear with respect to the angular frequency. More precisely, the solutions that are sought are quite different according to the value of the angular frequency. To underline this point, we have plotted in Fig. 9 the streamlines of the real part of the vector $\mathbf{V}$ for six values of the angular frequency. These figures show that the unknown vector evolves a lot with the angular frequency.This explains why more than 10 full steps are necessary to obtain the indicator curve with the RANM. Nevertheless, this number is relatively small compared to the number of steps required with the full model. As each step demands one matrix triangulation, which is the most time-consuming phase for the indicator calculus, one can then expect, with the RANM, a substantial decrease in the computational times (see Section ). Let us note that the matrix triangulation is carried out with a $L U$ decomposition.

According to the results presented in Table 5, one can give some tendencies on how the couple (nred, $\epsilon$ ) of the proposed reduced technique can be chosen. Hence, a small or moderate number of vectors $(10 \leq$ nred $\leq 30)$ have to be associated with a small value of $\epsilon\left(10^{-2} \leq \epsilon \leq 10^{-1}\right)$. Contrariwise, to a great number of vectors (nred $\geq 40$ ), one can link a great value for parameter $\epsilon(\epsilon \geq 1)$. Nevertheless, this analysis must be completed with 
a study of computational times.

\subsection{Computational times}

For this section, which is devoted to the study of the computational times, we consider the second example: the flow in a channel (Fig. 2). The evolution of the indicator versus the angular frequency is plotted in Fig. 10 for Mesh 3. In this figure, the indicator curve obtained with RANM is also plotted with nred $=30$ and $\epsilon=10^{-1}$. The curve is plotted for an angular frequency varying between 0 and 130 . The reference curve, with the full model, is obtained with nearly 50 steps of the continuation method. To study the computational times with the RANM, the value of the parameter $\epsilon$ is fixed and equal to $10^{-1}$, and the number of vectors, nred, varies between 20 and 40 (according to the discussion above). With these values, the RANM gives exactly the same number of Hopf bifurcation points as the full model and with approximately the same number of iterations of the Newton method. As for the lid-driven cavity, the number of steps required to get the indicator curve up to $\omega=130$ is greater with the RANM than with the full size model. Nevertheless, the number of full steps with the RANM, and for the considered number of vectors, is only about 15 (50 with the full model). With these results, therefore, the computational times needed to obtain the indicator curve can be estimated for all the methods that are considered. In Fig. 11, the cost of the RANM and of the full model are plotted according to the number of unknowns of the numerical example. These curves are 
obtained by considering the CPU times required for the full steps and also for building the reduced operators. These computational times are relative to the CPU times required for a single eigenvalues computation obtained with ARPACK. This computational time is then chosen as the reference time in this study.Fig. 11, therefore, shows that the indicator curve with the full model is obtained with a computational time which is about 0.5 times a single calculus with ARPACK for Mesh 1 and 4.5 times greater with Mesh 4. Let us recall that an indicator curve gives, for all the considered meshes, 4 or 5 initial guesses for the Newton method leading to 2 accurate Hopf bifurcation points. By comparison, with ARPACK, at least 4 calculi are needed to get an idea as to where the bifurcation occurs, even more if accurate critical values are required. With the Hybrid method (the RANM or the full model), accurate bifurcation points can be found even if the indicator curve is computed far from the critical Reynolds number. Hence, the first bifurcation point (for Mesh 1) is also determined if the indicator curve is computed at a Reynolds number equal to 160 (far from the critical number which is 975 in this example, see Table 3). This is a real advantage for the hybrid method compared to other types of numerical methods usually used to determine precise instability points. By considering the computational times obtained with the RANM in Fig. 11, one can see that this method required approximately 2 or 3 three times less times than the full model. The lowest computational times is normally obtained with the smaller number of vectors, nred $=20$ (see Fig. 11), and is equal to 1.5 times an ARPACK 
calculus. This number of vectors seems to be the optimum value leading to the lowest computational times with the same number of critical points being found.

Nevertheless, the computational times obtained with the RANM are still relatively important. Let us have a look in detail at the times required with the RANM. Hence in Fig. 12-a, we have plotted the evolution of the CPU times to construct the reduced matrix defined in Eq. 25 for the 4 considered meshes and for nred between 20 and 40. Theses times represent the projection and also the triangulation of the reduced matrix. The results presented in this figure show that the increase in computational times for the calculation of the reduced matrix is relatively important when the number of unknowns increases. Considering Mesh 4, the cost of a full matrix triangulation is nearly 14, 9 and 7 times the computational times of the reduced matrix respectively for nred $=20,30$ and 40 . This can explain why the CPU times are lower with nred $=20$, whereas with this value the number of full steps using RANM is greater than with other values of nred (see Table 5). Finally in Fig. 12-b, we have plotted the evolution of the computation of the reduced right-hand side $\mathcal{F}$ (see Eq. 25) versus the number of unknowns in the problem. As for the reduced matrix, several vector numbers in the basis are considered. The order of truncature of the perturbation method, p, is equal to 30. Firstly, this plot shows that the computational times for the reduced right-hand side do not depend on the considered nred. Secondly, as for the full size model (see results in Table 2), these computational times linearly increase with the 
number of unknowns. Thirdly, these times are relatively low compared to the times that are needed for the same quantities computed on the full size problem. In fact $17 \mathrm{~s}$ are required for Mesh 4 (nred=40) whereas 110s are needed for the same mesh on the full size model.

\section{Conclusion}

In this work, we have proposed a reduced order method to compute an indicator curve. On this curve, minima are automatically computed and introduced as initial values in a Newton iterative scheme. This permits to define an automatic method to compute Hopf bifurcation points for 2 dimensional fluid flows. The proposed method is very simple because it couples full size and reduced order steps. In fact, the indicator is computed by using a perturbation method whose solutions at each order of truncature, in the full size problem, are used to build the reduced basis. From this computation, several reduced steps are carried out to determine a supplementary part of the indicator curve. The proposed low-dimensional model requires two user parameters which are the number of vectors of the reduced method, nred, and an accuracy parameter, $\epsilon$. The numerical examples presented in this paper show that the reduced technique leads to the same results as the full model while requiring lower computional times (less than two ARPACK computations) if the two parameters $\epsilon$ and nred are respectively close to $10^{-1}$ and 20 . Finally, the RANM keeps the advantages of the hybrid method introduced in Ref. [1] and improved in Ref. [8] it means robust and automatic method to 
detect several Hopf bifurcation points for a knowledge of a single steady solution which can be far from the singular points. Moreover, with the reduced method presented in this paper, the detection of singular points can be carried out with low computational times, which is a real improvement of the hybrid method.

For other problems, for instance 3D problems, numerical tests have to be performed to find the optimal values in each case. As the proposed reduced model has been initially successfully evaluated for other kinds of nonlinear problems (see Ref. $[10,13,14]$ ), one can expect positive results in the 3D fluid mechanics framework.

The reduced model leads to computational times which are nearly three times lower than with the full size problems. A large part of these times are due to the construction of the reduced size matrices which become large when the number of unknowns is important. A possible way to reduce these times is to use an a priori hyperreduction method $[15,16]$. On the one hand, such method permits to decrease the computational times for the reduced method by selecting the most convenient data. On the other hand, the previous method is a learning method which enables us to improve the validity range of the reduced model. Hence, at the end of the reduced step, an updating basis step is carried out leading to an improvement of the basis. We are still working on this topic.

Finally with the proposed reduction method, the CPU times for the computation of the first phase of the Hybrid method (calculus of the indicator 
curve) is reduced. However, the second phase (Newton iterative method) also requires a lot of computation times which can be reduced by using a highorder iterative corrector [13] or by using convergence acceleration methods [19] which make it possible to decrease the number of Newton iterations.

\section{References}

[1] A. Brezillon, G. Girault and J.M. Cadou, A numerical algorithm coupling a bifurcating indicator and a direct method for the computation of Hopf bifurcation points in fluid mechanics, Computers \& Fluids, 2010, $39,1226-1240$

[2] A. Fortin, M. Jardak, J.J. Gervais and R. Pierre, Localization of Hopf bifurcations in fluid flow problems, International Journal for Numerical Methods in Fluids, 1997, 24, 1185-1210.

[3] V.B.L. Boppana and J.S.B. Gajjar, Global flow instability in a lid-driven cavity, International Journal for Numerical Methods in Fluids, 2010, $62(8), 827-853$.

[4] C. P. Jackson, A finite-element study of the onset of vortex shedding in flow past variously shaped body, Journal of Fluid Mechanics, 1987, 182, $23-45$.

[5] A. D. Jepson, Numerical Hopf bifurcation, Thesis, California Institute of technology, 1981. 
[6] R. Seydel, From equilibrium to chaos, Practical Bifurcation and stability analysis. Springer-Verlag New-York, 1994.

[7] J.M. Cadou, M. Potier-Ferry and B. Cochelin, A numerical method for the computation of bifurcation points in fluid mechanics, European Journal of Mechanics B/Fluids, 2006, 25, 234-254.

[8] G. Girault, Y. Guevel and J.M. Cadou, An algorithm for the computation of multiple Hopf bifurcation points based on Padé approximants, International Journal for Numerical Methods in Fluids, 2012, 68, 11891206.

[9] M. Medale, B. Cochelin, A parallel computer implementation of the Asymptotic Numerical Method to study thermal convection instabilities, Journal of Computational Physics, 2009, 228(22), 8249-8262.

[10] J.M. Cadou and M. Potier-Ferry, A solver combining reduced basis and convergence acceleration with applications to non-linear elasticity, International Journal for Numerical Methods in Biomedical Engineering, 2010, 26, 1604-1617.

[11] Lumley J.L., The structure of inhomogeneous turbulence, Atmospheric Turbulence and Wave Propagation, Ed. A.M. Yaglom \& V.I. Tatarski, 1967.

[12] W. Cazemier, R.W.C.P. Verstappen and A.E.P. Veldman, Proper or- 
thogonal decomposition and low-dimensional models for driven cavity flows, Physics of Fluids, 1998, 10-7, 1685-1699.

[13] J.-M. Cadou and N. Damil and M. Potier-Ferry and B. Braikat, Projection techniques to improve high-order iterative corrector, Finite Element in Analysis and Design, 2004, 41, 285-309.

[14] F. Boumediene, L. Duigou, E.H. Boutyour, A. Miloudi, J.M. Cadou, Nonlinear forced vibration of damped plates coupling Asymptotic Numerical Method and reduction models, Computational Mechanics, 2011, 47(4), 359-377

[15] D. Ryckelynck, L. Hermanns, F. Chinesta and E. Alarcon, An efficient a priori model reduction for boundary element models, Engineering Analysis with Boundary Elements, 2005, 29, 796-801.

[16] C. Allery, A. Hamdouni, D. Ryckelynck, N. Verdon, A priori reduction method for solving the two-dimensional Burgers equations. Applied Mathematics and Computation, 2011, 217, 15,6671-6679.

[17] J.M. Cadou, B. Cochelin, N. Damil and M. Potier-Ferry, Asymptotic Numerical method for stationary Navier-Stokes equations and with PetrovGalerkin formulation, International Journal for Numerical Method in Engineering, 2001, 50, 825-845.

[18] B. Cochelin, A path-following technique via an asymptotic-numerical method, Computers \& Structures, 1994, 53, 5, 1181-1192. 
[19] J.M. Cadou, L. Duigou, N. Damil, M. Potier-Ferry, Convergence acceleration of iterative algorithms. Applications to thin shell analysis and Navier Stokes equations, Computational Mechanics, 43-2 (2009) 253264.

[20] O.C. Zienkiewicz and R.L. Taylor, The Finite Element Method, 2, McGraw-Hill Book Company, 4th Edition, 1991.

[21] Y. Guevel, H. Boutyour, J.M. Cadou, Automatic detection and branch switching methods for steady bifurcation in fluid mechanics, Journal of Computational Physics, 2011, 230, 9, 3614-3629.

[22] G.A. Baker and P. Graves-Morris, Padé approximants, Encyclopedia of Mathematics and its applications, Cambridge University Press,Cambridge, Second edition, 1996.

[23] A. Elhage-Hussein, M. Potier-Ferry and N. Damil, A numerical continuation method based on Padé approximants, International Journal of Solids and Structures, 2000, 37, 6981-7001.

[24] A. Najah, B. Cochelin, N. Damil and M. Potier-Ferry, A critical review of asymptotic numerical methods, Archives of Computational Methods in Engineering, 1998, 5, 3-22.

[25] J. Mizushima, H. Okamoto and H. Yamaguchi, Stability of flow in a channel with a suddently expanded part, Physics of Fluids, 1996, 8(11), 2933-2942. 
[26] M. Poliashenko and C.K. Aidun, A direct method for computation of simple bifurcations, Journal of Computational Physics, 1995, 121, 246260.

[27] C.H. Bruneau and M. Saad, The 2D lid-driven cavity problem revisited, Computers \& Fluids, 2006, 35, 326-348.

[28] Y.F. Peng, Y.H. Shiau and R. R. Hwang, Transition in 2-D lid-driven cavity flow, Computers \& Fluids, 2003, 32, 337-352.

[29] A. Abouhamza and R. Pierre, A neutral stability curve for incompressible flows in a rectangular driven cavity, Mathematical and Computer Modelling, 2003, 38, 141-157.

[30] J.J. Gervais, D. Lemelin and R. Pierre, Some experiments with stability analysis of discrete incompressible flows in the lid-driven cavity, International Journal for Numerical Methods in Fluids, 1997, 24, 477-492.

[31] G. Tiesinga, F.W. Wubs and A.E.P. Veldman, Bifurcation analysis of incompressible flow in a driven cavity by the Newton-Picard method, Journal of Computational and Applied Mathematics, 2002, 140, 751772 .

[32] F. Auteri, N. Parolini and L. Quartapelle, Numerical investigation on the stability of singular driven cavity flow, Journal of Computational Physics, 2002, 183, 1-25. 
[33] R. Lehoucq, D.C. Sorensen and C. Yang, Arpack User's Guide: Solu$\underline{\text { tion of large-scale eigenvalue problems with implicitly restarted Arnoldi }}$ methods, SIAM, Philadelphia, 1998.

[34] C. Allery, J.M. Cadou, A. Hamdouni and D. Razafindralandy, Application of the Asymptotic Numerical Method to the Coanda effect study, Revue Européenne des Eléments Finis, 2004, 13, 57-77.

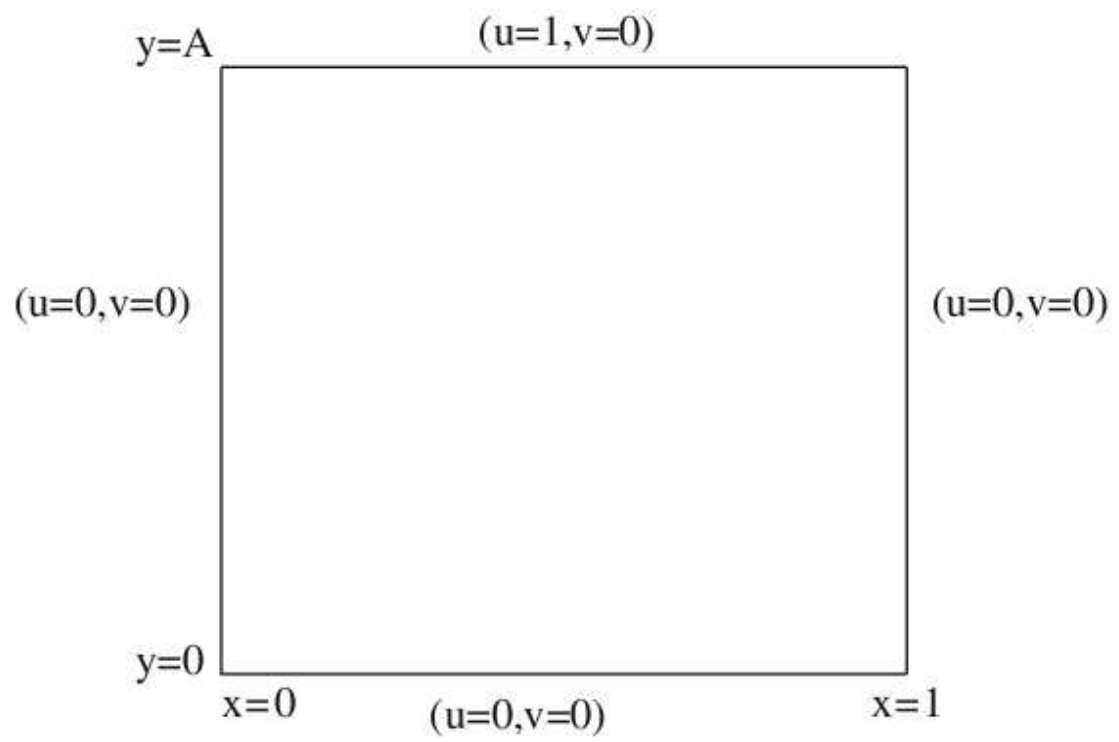

Figure 1: Geometry for the two-dimensional lid-driven cavity flow (case $A=1$ ). 


\begin{tabular}{|c|c|c|c|}
\hline Authors & $\operatorname{Re}_{\mathrm{c}}$ & $\mathrm{St}_{\mathrm{c}}$ & $\omega_{c}$ \\
\hline Brezillon et al.[1] & 7890 & 0.44 & 2185 \\
\hline Fortin et al. [2] & 8000 & 0.45 & 2261.9 \\
\hline Boppana and Gajjar[3] & 8026.6 & 0.4497 & 2268.0 \\
\hline \multirow{2}{*}{ Cazemier et al.[12] } & 7819 & 0.61 & 2996.8 \\
\hline & 7972 & 0.45 & 2254.0 \\
\hline Polishenko and Aidun[26] & 7763.4 & 0.45 & 2195.0 \\
\hline Bruneau and Saad[27] & $8000-8050$ & - & - \\
\hline Peng et al. [28] & 7402 & 0.59 & 2744.0 \\
\hline Abouhamza and Pierre[29] & 8004.5 & - & - \\
\hline \multirow{2}{*}{ Gervais et al. $[30]$} & 7960 & 0.45 & 2250.6 \\
\hline & 8040 & 0.45 & 2273.3 \\
\hline Tiesinga et al.[31] & 8375 & 0.4399 & 2314.8 \\
\hline Auteri et al.[32] & $\approx 8018$ & 0.4496 & 2265 \\
\hline Present study (13122 d.o.f.) & 7890 & 0.44 & 2185 \\
\hline
\end{tabular}

Table 1: Comparison of the critical parameters for the first Hopf bifurcation from the literature. The considered example is the lid-driven cavity with $\mathrm{A}=1$.

\begin{tabular}{lcccc}
\hline & & & \multicolumn{3}{c}{ CPU times for indicator (s) } \\
Name & Number of d.o.f. & ARPACK CPU times $(s)$ & $\mathbf{K}_{t}($ Eq. 15) & $\mathbf{F}_{p}($ Eq. 15) \\
\hline Mesh 1 & 32.322 & 575 & 3.5 & 4.5 \\
Mesh 2 & 127.042 & 2500 & 58 & 28 \\
Mesh 3 & 261.890 & 5160 & 313 & 80 \\
Mesh 4 & 347.462 & 6950 & 560 & 110 \\
\hline
\end{tabular}

Table 2: Number of degree of freedom used for the example of the flow in a channel (Fig. 2). The order of truncature for the computation of the right hand side $\mathbf{F}_{p}$ and the unknown vectors $\mathbf{V}_{p}$ is equal to 30 . 


\begin{tabular}{ll|ccc|ccc|}
\hline & & \multicolumn{5}{|c}{ Hopf bifurcation, critical parameters. } \\
& & $\mathrm{Re}_{c} 1$ & $\omega_{c 1}$ & $\mathrm{St}_{1}$ & $\mathrm{Re}_{c} 2$ & $\omega_{c 2}$ & St $_{2}$ \\
\hline \multirow{3}{*}{ Present study } & Mesh 1 & 975 & 46.74 & 0.076 & 1578 & 103.16 & 0.104 \\
& Mesh 2 & 988 & 47.03 & 0.075 & 1533 & 99.93 & 0.103 \\
& Mesh 3 & 993 & 47.24 & 0.075 & 1538 & 100.18 & 0.103 \\
& Mesh 4 & 994 & 47.3 & 0.075 & 1539 & 100.28 & 0.103 \\
\hline ARPACK & Mesh 1 & {$[965,1023]$} & $\approx 47$ & - & {$[1559,1602]$} & $\approx 103$ & - \\
\hline Mizushima et al. $[25]$ & - & 843 & & 0.074 & - & - & - \\
\hline
\end{tabular}

Table 3: Hopf bifurcation points for the example of the flow in a channel (Fig. 2), A =7/3.

\begin{tabular}{ccc|ccccc}
\hline \multicolumn{6}{c|}{ Initial values } & \multicolumn{6}{c}{ Critical values } \\
$\operatorname{Re}_{i}$ & $\omega_{i}$ & $\Phi_{i}$ & $\mathrm{Re}_{c}$ & $\omega_{c}$ & $\mathrm{St}_{c}$ & Number of iterations & $\mathrm{N}^{\circ}$ \\
\hline & 754 & 0.64 & - & - & - & - & \\
& 1085 & 0.606 & - & - & - & - & \\
& 1360 & 0.418 & - & - & - & - & 6 \\
& 1759 & 0.486 & 11286 & 2385 & 0.33 & 6 & 2 \\
8089 & 2236 & 0.395 & 7890 & 2185 & 0.44 & 5 & 3 \\
& 2691 & 0.669 & 8829 & 2925 & 0.52 & 5 & 4 \\
& 3105 & 1.234 & 8921 & 3424 & 0.61 & 7 & 5 \\
\hline
\end{tabular}

Table 4: Numerical results from Ref. [8] obtained for the problem of the lid-driven cavity - case A=1.0 - Critical Reynolds and Strouhal numbers obtained for initial Reynolds numbers evaluated as minima of the indicator curve. Symbol '-' stands for the non convergence of the Newton method. 


\begin{tabular}{|c|c|c|c|c|c|c|c|c|c|}
\hline & Order $p$ & $\epsilon$ & N1 & $\mathrm{N} 2$ & $\mathrm{Nr}$ & $\mathrm{NH}$ & N3 & $\mathrm{NT}$ & $\mathrm{N}^{\circ}$ \\
\hline \multirow{3}{*}{ Full model } & 20 & - & 76 & 0 & 10 & 5 & 32 & 76 & All \\
\hline & 25 & - & 69 & 0 & 12 & 6 & 36 & 69 & All \\
\hline & 30 & - & 66 & 0 & 9 & 6 & 36 & 66 & All \\
\hline \multirow{3}{*}{ nred $=10$} & 30 & $10^{-2}$ & 83 & 57 & 13 & 8 & 46 & 26 & $1,2,3,3,4,5,6,6$ \\
\hline & 30 & $10^{-1}$ & 70 & 53 & 13 & 7 & 43 & 17 & $1,2,3,4,5,6,6$ \\
\hline & 30 & 1 & 39 & 33 & 5 & 3 & 20 & 6 & $1,2,4$ \\
\hline \multirow{3}{*}{ nred $=20$} & 30 & $10^{-2}$ & 78 & 56 & 10 & 6 & 36 & 22 & All \\
\hline & 30 & $10^{-1}$ & 68 & 52 & 10 & 6 & 36 & 16 & All \\
\hline & 30 & 1 & 31 & 27 & 3 & 1 & 5 & 4 & 2 \\
\hline \multirow{4}{*}{ nred $=25$} & 30 & $10^{-2}$ & 77 & 54 & 10 & 6 & 37 & 23 & All \\
\hline & 30 & $10^{-1}$ & 73 & 56 & 10 & 6 & 36 & 17 & All \\
\hline & 30 & 1 & 59 & 46 & 10 & 6 & 37 & 13 & All \\
\hline & 30 & 10 & 20 & 17 & 2 & 0 & - & 3 & - \\
\hline \multirow{4}{*}{ nred $=30$} & 30 & $10^{-2}$ & 75 & 51 & 10 & 6 & 36 & 24 & All \\
\hline & 30 & $10^{-1}$ & 74 & 54 & 10 & 6 & 38 & 20 & All \\
\hline & 30 & 1 & 57 & 42 & 7 & 5 & 29 & 15 & $1,2,3,4,5$ \\
\hline & 30 & 10 & 19 & 16 & 2 & 0 & 0 & 3 & - \\
\hline \multirow{5}{*}{ nred $=40$} & 20 & $10^{-2}$ & 76 & 54 & 9 & 5 & 29 & 22 & $1,2,3,4,5$ \\
\hline & 30 & $10^{-1}$ & 73 & 55 & 10 & 6 & 36 & 18 & All \\
\hline & 30 & 1 & 67 & 52 & 10 & 6 & 36 & 15 & All \\
\hline & 30 & 10 & 70 & 48 & 11 & 5 & 28 & 22 & $1,2,3,4,5$ \\
\hline & 30 & $10^{2}$ & 69 & 52 & 12 & 6 & 36 & 17 & All \\
\hline \multirow{5}{*}{ nred $=50$} & 25 & $10^{-2}$ & 75 & 53 & 10 & 6 & 39 & 22 & All \\
\hline & 30 & $10^{-1}$ & 74 & 53 & 10 & 6 & 36 & 21 & All \\
\hline & 30 & 1 & 74 & 57 & 11 & 6 & 37 & 17 & All \\
\hline & 30 & 10 & 76 & 62 & 12 & 7 & 42 & 14 & $1,2,3,3,4,5,6$ \\
\hline & 30 & $10^{2}$ & 72 & 60 & 14 & 7 & 42 & 12 & $1,2,3,3,4,5,6$ \\
\hline \multirow{5}{*}{ nred $=60$} & 30 & $10^{-2}$ & 75 & 53 & 10 & 6 & 36 & 22 & All \\
\hline & 30 & $10^{-1}$ & 75 & 55 & 12 & 7 & 43 & 20 & $1,2,3,3,4,5,6$ \\
\hline & 30 & 1 & 73 & 56 & 10 & 6 & 36 & 17 & All \\
\hline & 30 & 10 & 70 & 56 & 10 & 6 & 37 & 14 & All \\
\hline & 30 & $10^{2}$ & 70 & 58 & 11 & 8 & 51 & 12 & $1,2,3,3,4,5,6,6$ \\
\hline
\end{tabular}

Table 5: The indicator curve is computed for a Reynolds number equal to 8089, $\omega_{\max }=4100, \delta=10^{-6}$. $\mathrm{N} 1, \mathrm{~N} 2, \mathrm{Nr}, \mathrm{NH}$ and N3 stand respectively for the number of steps, the number of reduced steps, the number of roots found, the number of Hopf bifurcation points determined and the total number of iterations in the Newton scheme. $\epsilon$ is the accuracy required in the reduced computation (see Eq. 27). NT is the number of full triangulated matrices. $\mathrm{N}^{\circ}$ is the number of Hopf bifurcation point defined in Table 4. 'All' means that the six singular values (Table 4) have been found. 


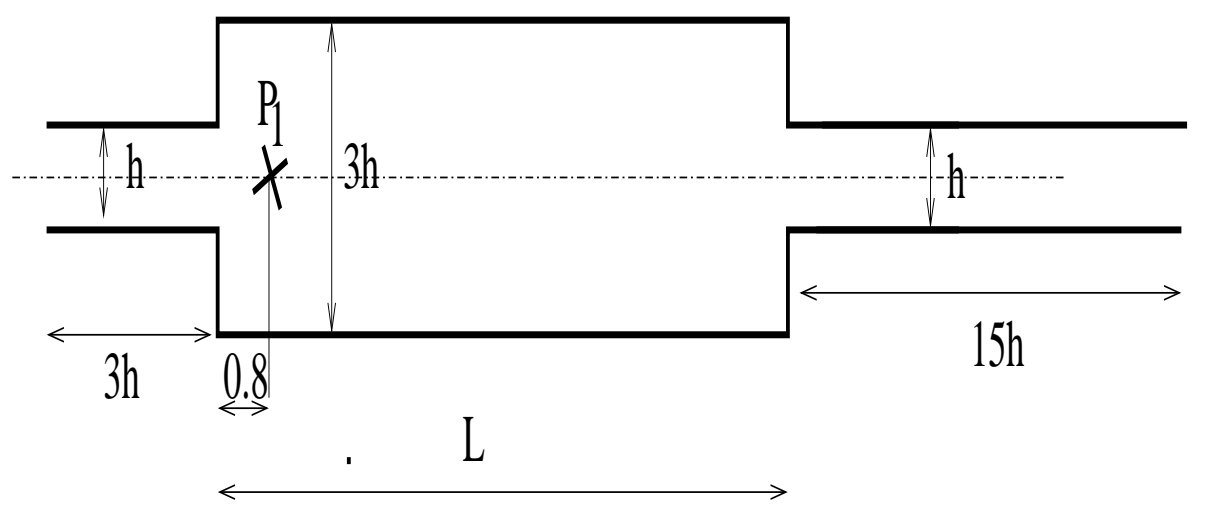

Figure 2: Channel geometry from Ref. [25], $\mathrm{A}=\frac{L}{3 h}$.

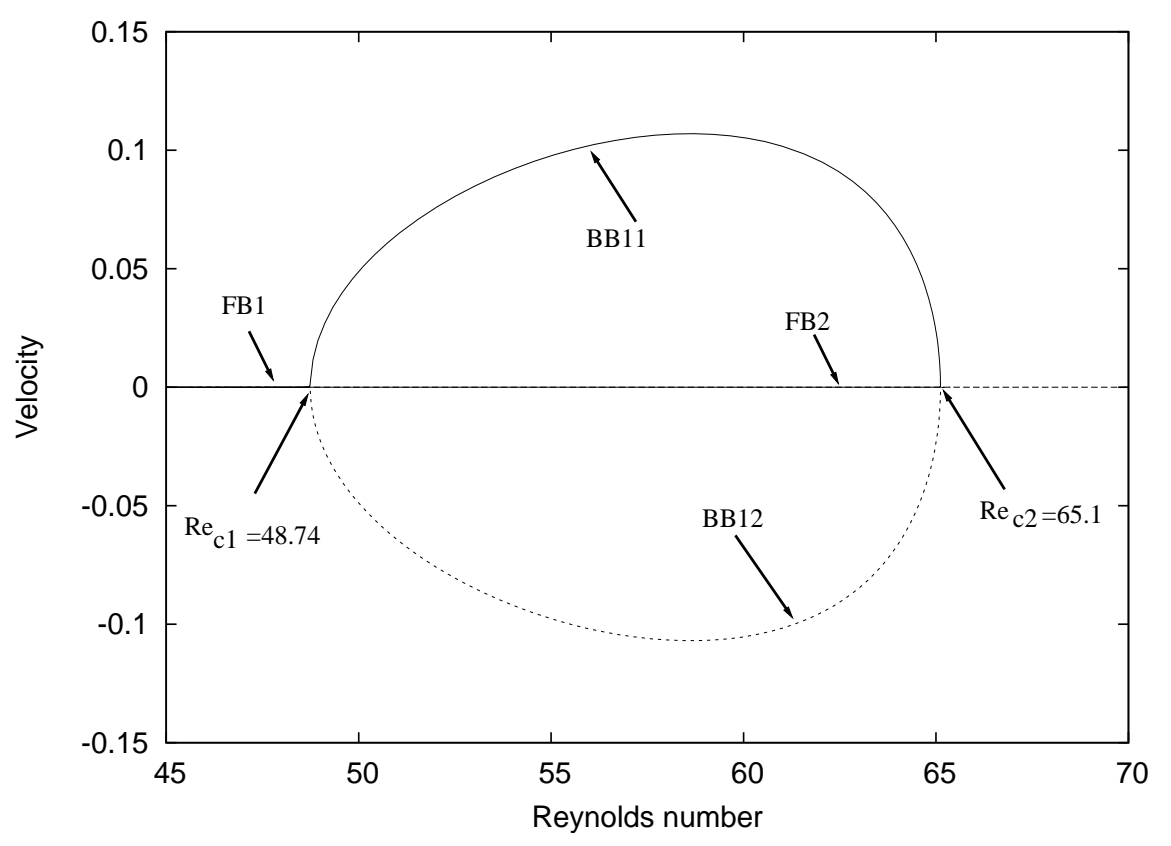

Figure 3: Velocity $u_{y}$ at point P1 (Fig. 2) versus the Reynolds number. Bifurcated branches and critical Reynols number from Ref. [21]. Flow in a channel[25], case A=7/3. 


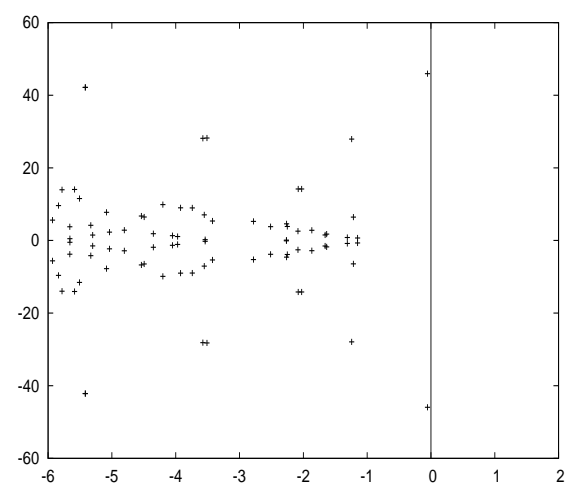

(a) $\operatorname{Re}=965$

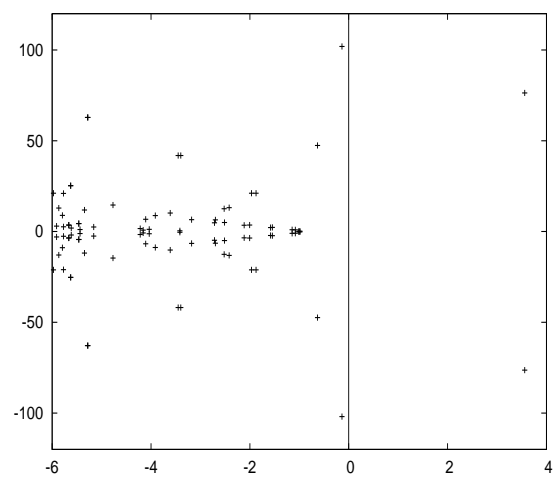

(c) $\operatorname{Re}=1559$

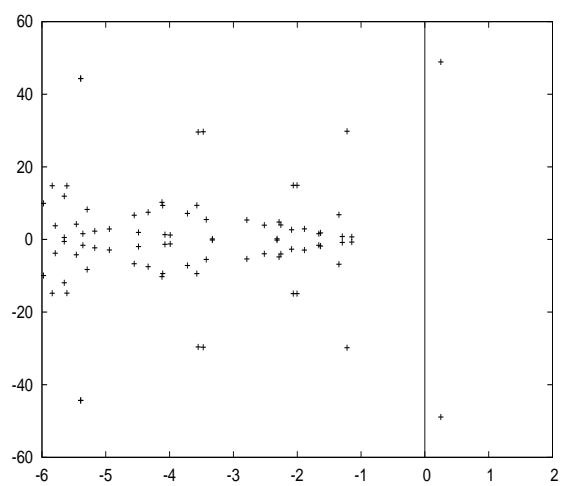

(b) $\operatorname{Re}=1023$

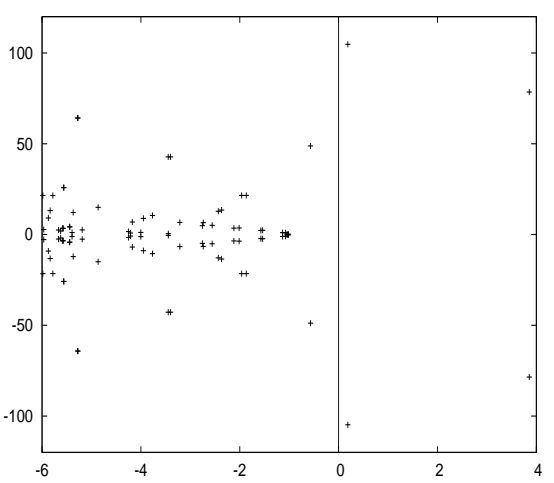

(d) $\operatorname{Re}=1602$

Figure 4: Eigenvalues in the complex plane. Example of the flow in a channel (Fig. 2), Mesh 1, $\mathrm{A}=7 / 3$. The critical parameters for this example are $\mathrm{Re}_{c 1}=975$ and $\omega_{c 1}=46.47$ and $\operatorname{Re}_{c 2}=1578$ and $\omega_{c 2}=103.16$ respectively for the first and second Hopf bifurcation point. 


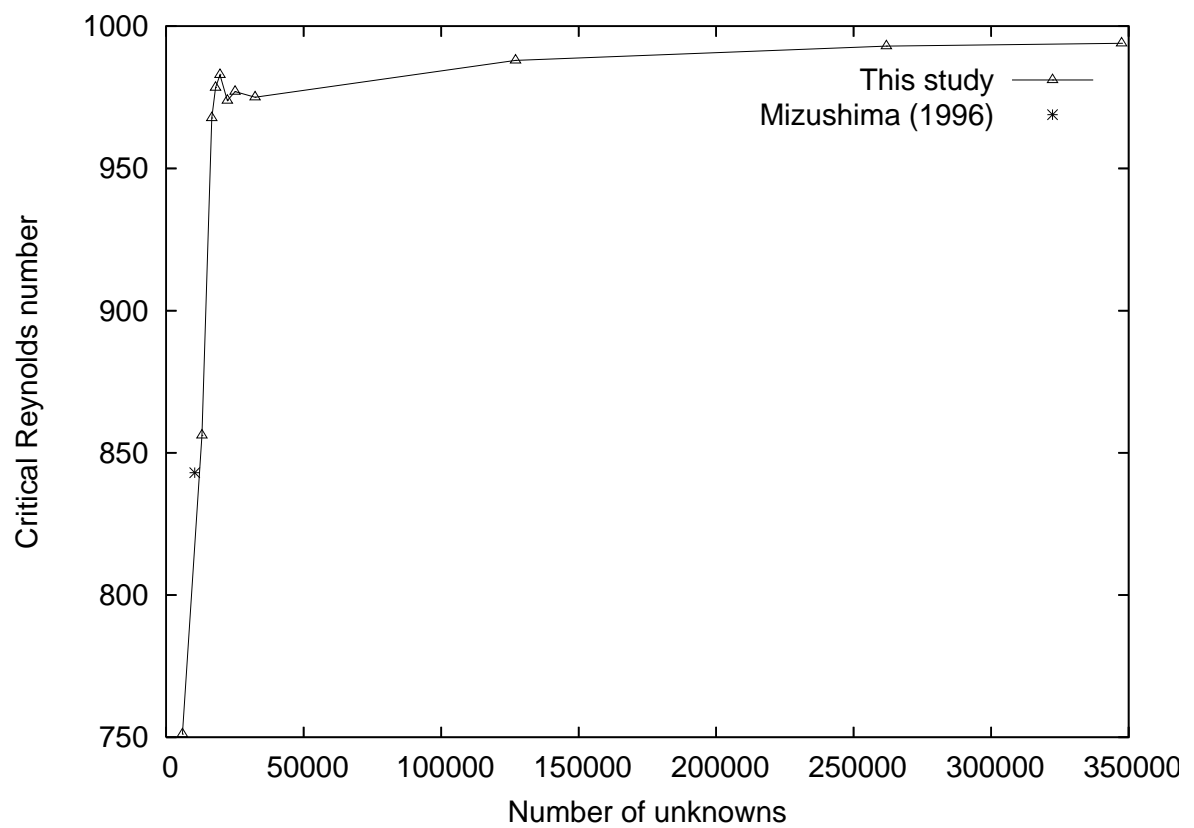

Figure 5: First critical Reynolds number versus the number of unknowns. Example of the flow in a channel (Fig. 2), $\mathrm{A}=7 / 3$. 


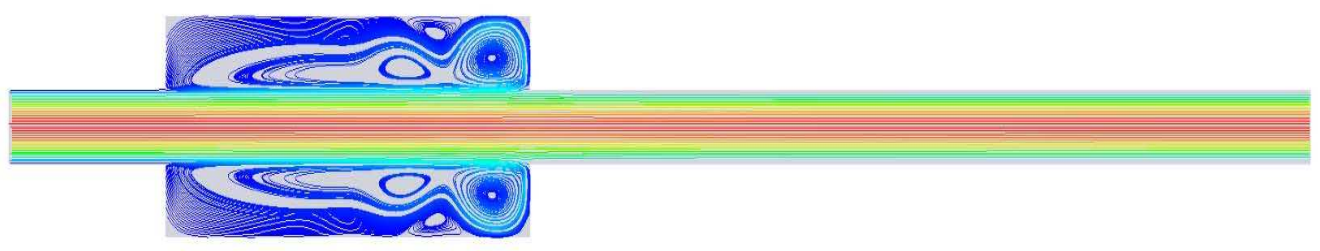

(a) Steady solution, $U^{S}$.

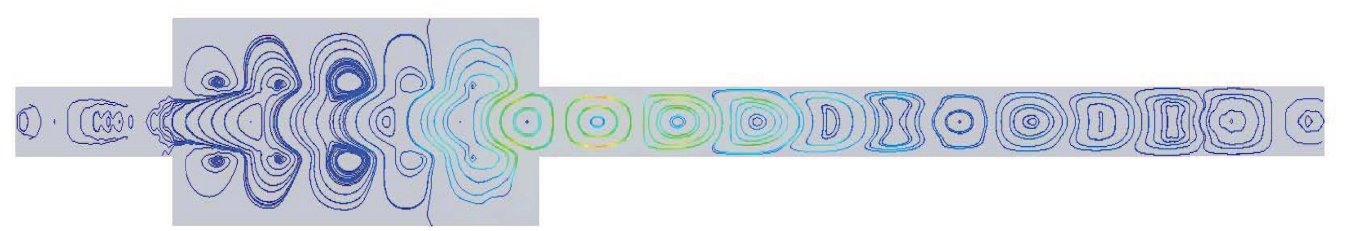

(b) Real eigenmode, $V^{R}$.

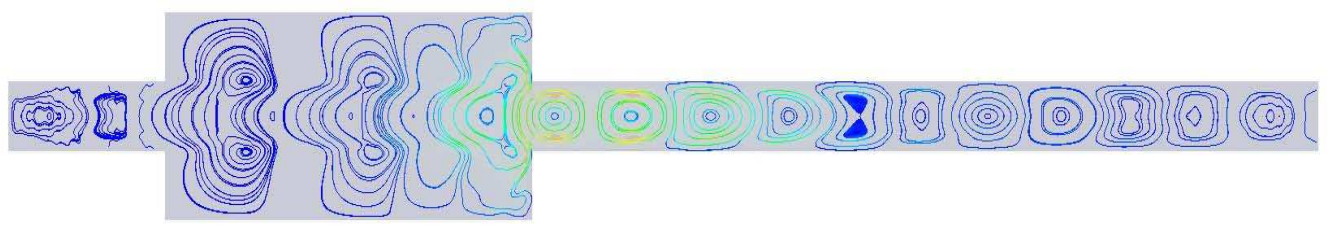

(c) Imaginary mode, $V^{I}$.

Figure 6: Streamlines of the velocity fields at the first critical Reynolds number, $\operatorname{Re}_{c 1}=856$ and $\omega_{c 1}=40.29$. Example of the flow in a channel (Fig. 2), $A=7 / 3,13000$ d.o.f. 


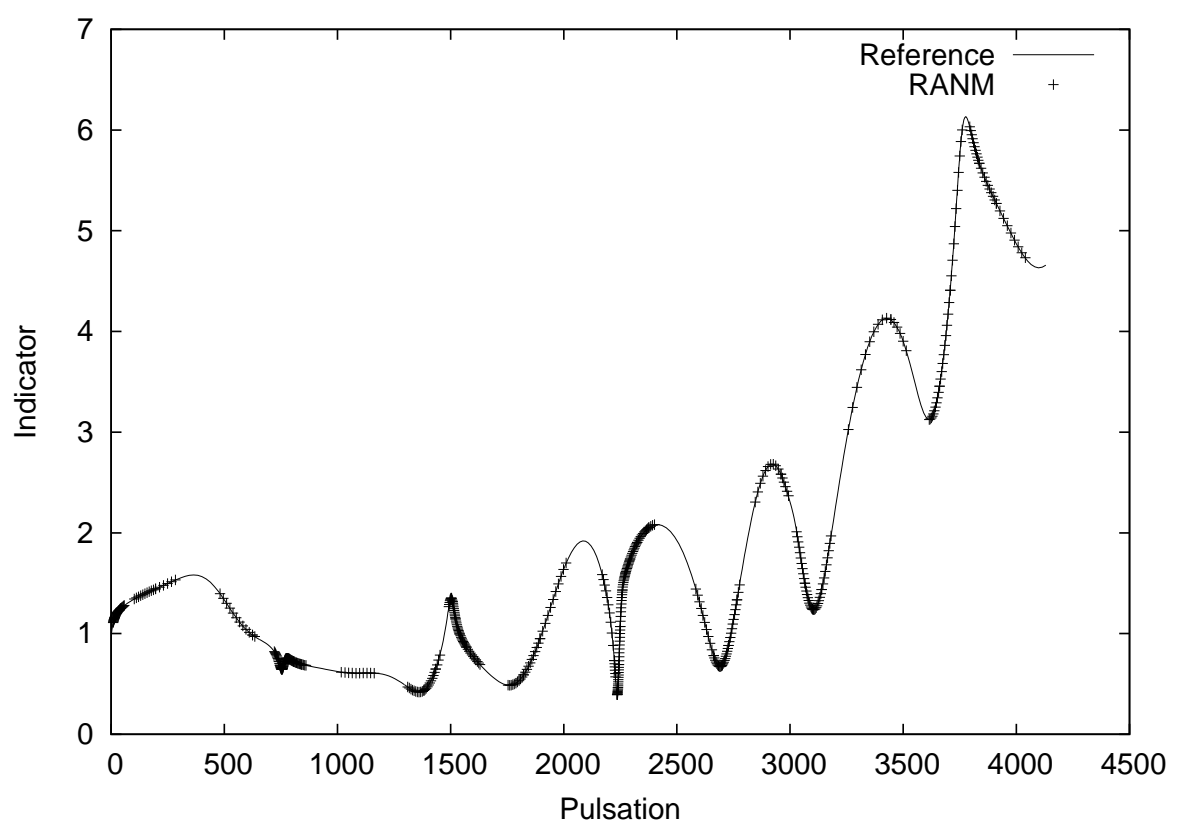

Figure 7: Indicator versus angular frequency at $\mathrm{Re}=8089$. Problem of the lid-driven cavity - case $A=1.0$. Order 30, nred $=60, \epsilon=10^{\circ}$. 


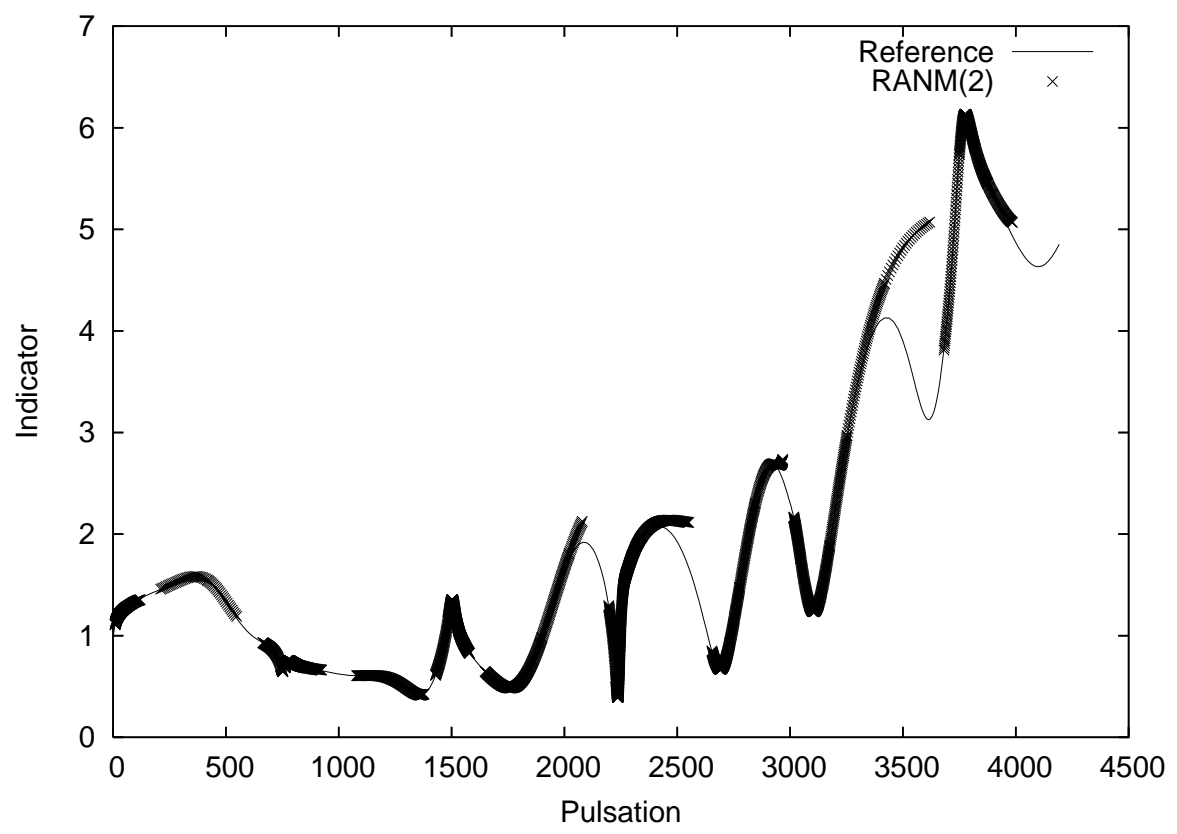

Figure 8: Indicator versus angular frequency at $\mathrm{Re}=8089$. Problem of the lid-driven cavity - case $\mathrm{A}=1.0$. $\operatorname{RANM}(2)$ stands for $\epsilon=10^{2}$. Order 30 and nred $=60$. 

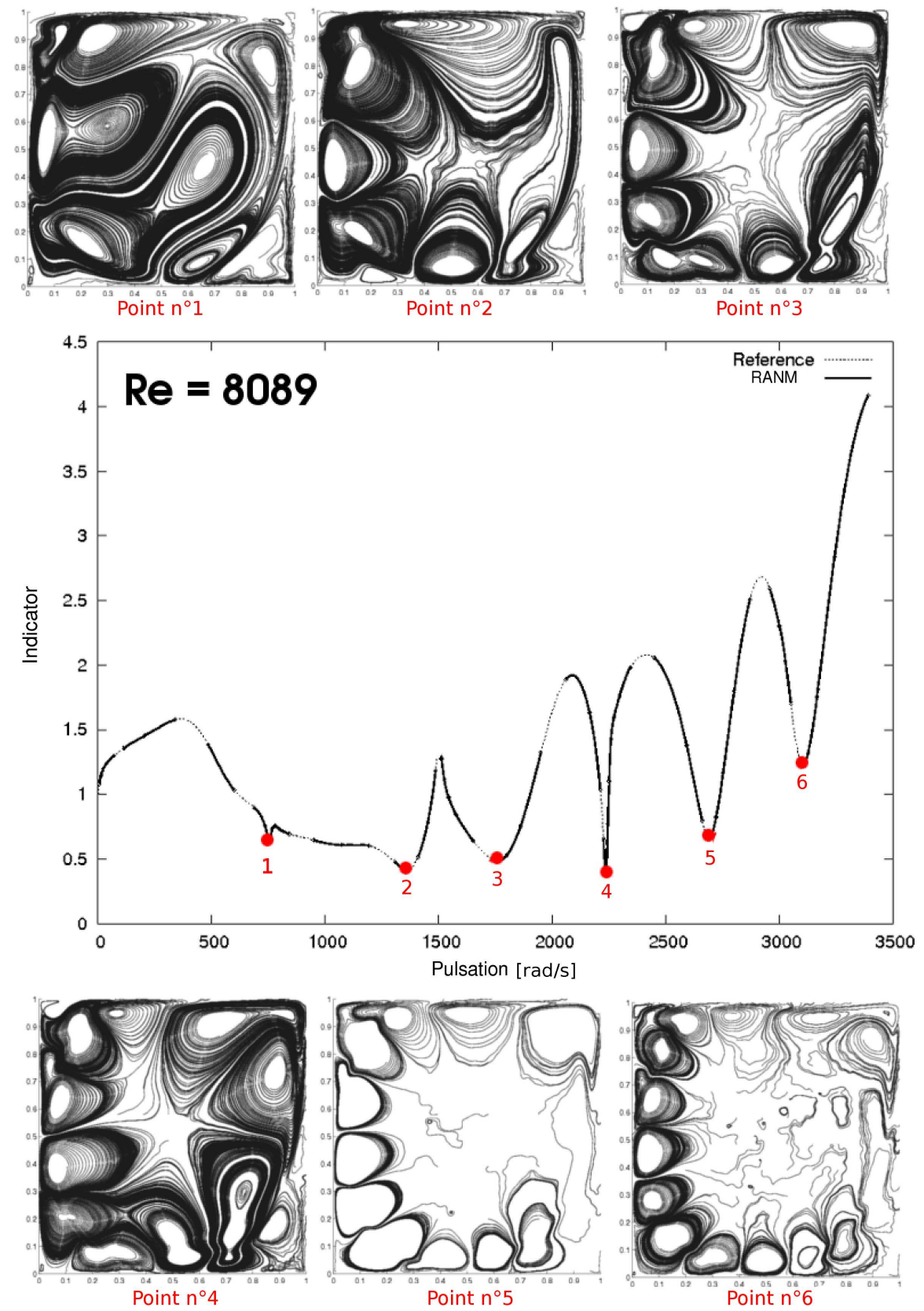

Figure 9: Indicator curve at $R e=8089$. Representation of the different initial values introduced in the Newton method. Problem of the lid-driven cavity - case A=1.0 


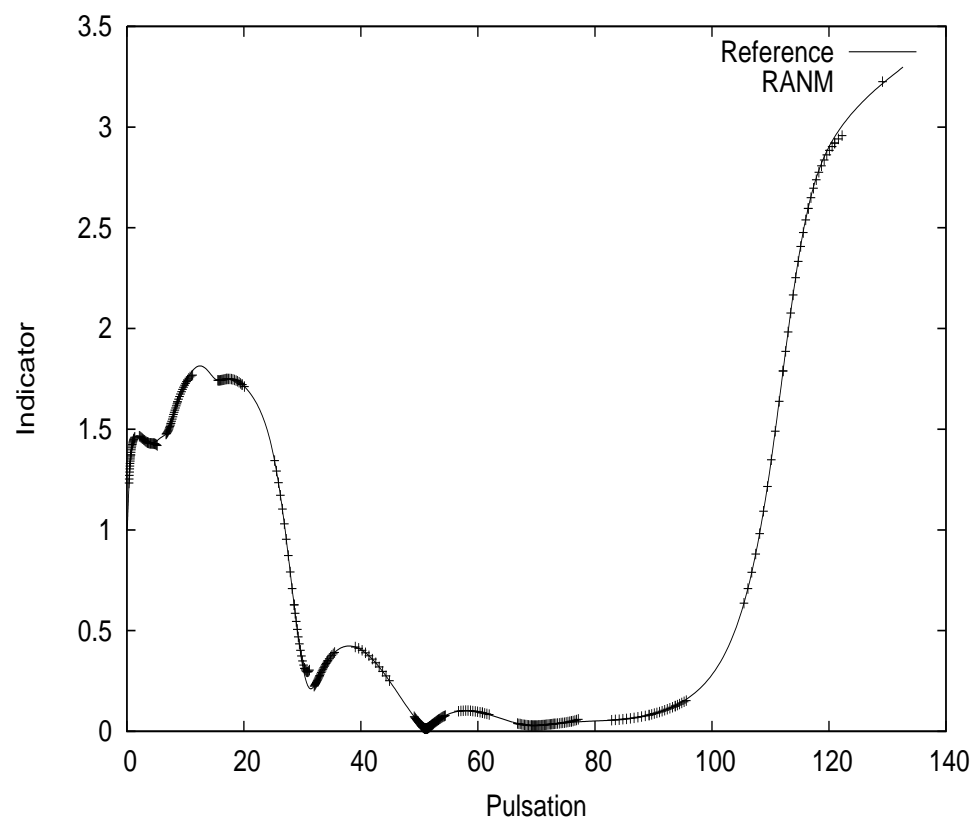

Figure 10: Indicator versus angular frequency for $R e=1068$. Example of the flow in a channel (Fig. 2), Mesh 3, $\mathrm{A}=7 / 3$. nred $=30$ and $\epsilon=10^{-1}$. 


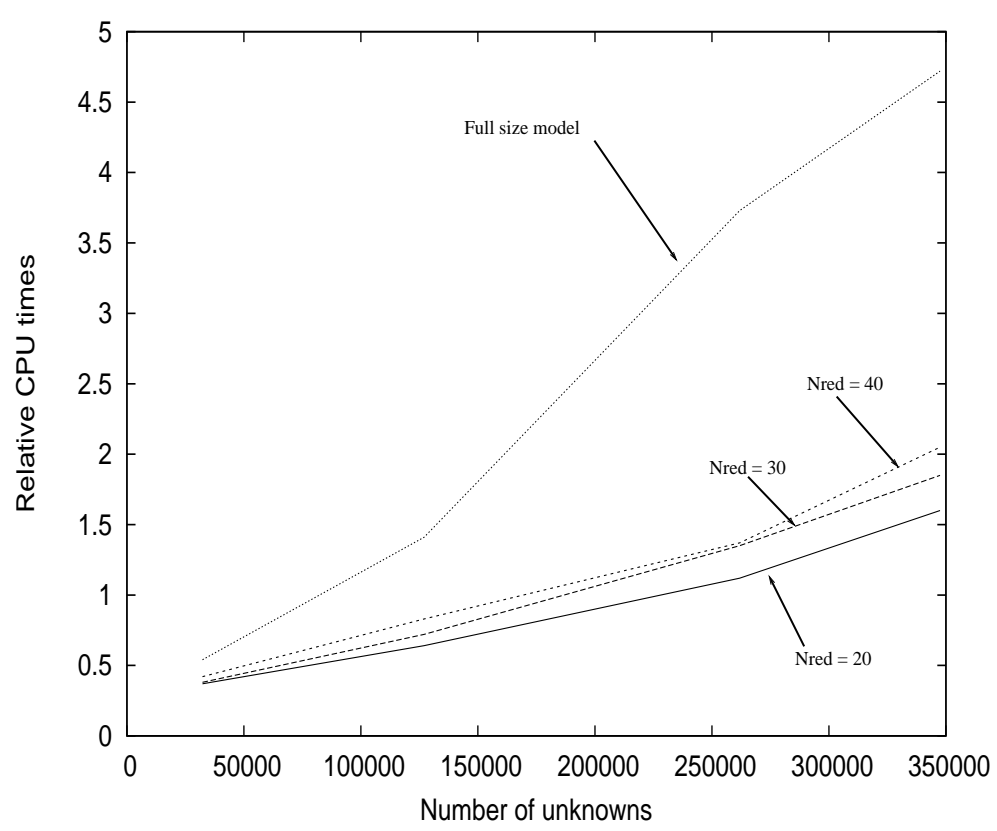

Figure 11: Cost of the reduced order models relative to a single ARPACK calculus. Example of the flow in a channel (Fig. 2), for the 4 meshes of Table 2.

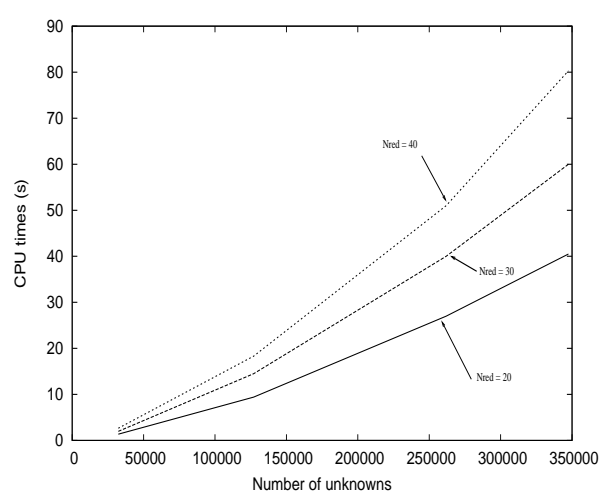

(a) Computation and triangulation of the reduced matrix $\mathcal{K}$ (Eq. 22).

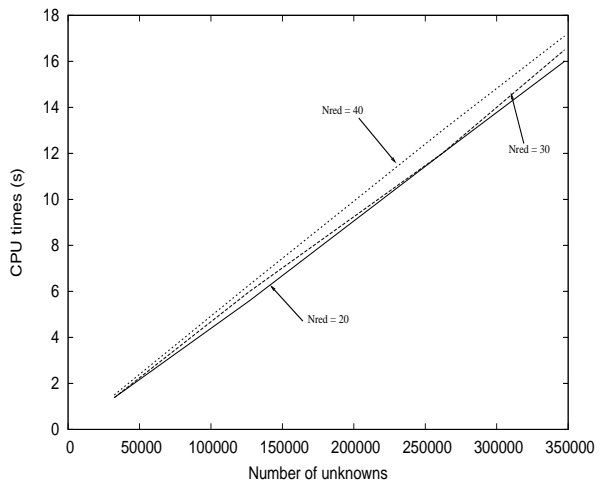

(b) Computation of the reduced right hand side $\mathcal{F}$ and unknown vector $\mathbf{v}$ (Eq. 22).

Figure 12: Evolution of the CPU times versus the number of unknowns of the problem. Example of the flow in a channel (Fig. 2). The order of truncature of the asymptotic expansions is equal to 30 . 